\title{
OBJECT-ORIENTED MAPPING APPLICATION OF ARCHITECTURE BASED ON MULTI-SCALE IMAGE SEGMENTATION AND IMAGE EXTRACTION
}

\author{
X. Qi *, X. Chen \\ ShenZhen Municipal Information Center of Land Resource, Urban Planning and Real Estate, 8007 Hongli Road \\ Shenzhen, P.R.China \\ rosexuanqi@gmail.com;xueye31@163.com
}

Commission ICWG (IV/II/VIII)

KEY WORDS: Mapping, Architecture, Multi-scale segmentation, Object-oriented, Extraction

\begin{abstract}
:
Residential area detection is extremely important for supervision of rush-built and illegal construction behavior to protect arable land and basic farmland. Traditional manual recognition of illegal construction is simple but time-consuming. The specific research work in the thesis includes: the proposition and designing of a multi-scale segmentation method based on watershed segmentation combined with region merging algorithm, comparative experiments of the new algorithm and traditional algorithm. Then, architecture extraction experiment based on the multi-scale segmentation method is carried out and analysis of the precision of comparative experiment results of pixel-based and object-oriented extraction methods is made, all these steps facilitate the automatic mapping of the architecture.
\end{abstract}

\section{INTRODUCTION}

Urban land ministry requires timely acquisition and analysis of spatial and temporal information for making informed decisions on land utilization and land supervision. As for Shenzhen, rapid economic development and accelerating urbanization process lead to excessive rush-built behavior and illegal construction. The new illegal buildings count up to 6.075 million square meters in 2011, compared with 9.871 million square meters in 2010. Present practice for mapping illegal buildings purely depends on manual visual interpretation, which is time and labor consuming. Object extraction method brings similar pixels into the same feature space according to spectral information and spatial information. In this way, buildings do not need to be drawn respectively through digging the whole high-resolution satellite image, they can be extracted into several categories, such as permanent ones, temporary ones, independent ones, continuous ones according to different spectral, shape, texture, topological attributes.

High-resolution satellite image is different from mediumresolution in many aspects, taking several examples, volume of data increased hugely with the improvement of resolution, spatial computation is increased consequently, image contains more intensive and abundant geographical and terrain information, the shadow of features is more evident. Urban land ministry tends to high-resolution satellite image to gain more accuracy in land changing investigation and illegal building investigation.

Image segmentation is a technique to divide images into regions with different features. The regions do not intersect with each other and features belonging to different regions have the largest divergence, but within a region features have similar attributes. Advance in image segmentation include four aspects: threshold segmentation, edge detection segmentation, regionbased segmentation and segmentation based on specific characteristics. But there are drawbacks for these methods, over-segmentation and under-segmentation are common for incorrect scale. It's difficult to transform segmentation results of various scales. Multi-scale segmentation refers to a polygon object hierarchical network technology with smallest heterogeneity and largest homogeneity. Each object level has fixed scale, and multiple object layers reflect attributes of various space objects. In such system, attributes for single image object contain relationship with adjacent objects, child objects and father objects. Object-oriented segmentation is a hot topic, which includes the FNEA method introduced by Definiens software. But the FNEA method practices original segmentation from pixel level, so it has complex computation, simultaneously, it is difficult to extract consistent border. Watershed segmentation method divides the gradient of image into target and background, the gradient as a whole landform surface, the target is a relatively homogeneous area inside with lower gradient, target has minimum gray value points. When the water in different zones is rising to meet the border, the dam formed the watershed line. Improved watershed segmentation puts pixels in the increasing order of pixel gray value and fills the queue by quick pixel scanning with width priority. Then, image is divided into larger areas with high internal heterogeneity. Region Adjacency Graph is composed of regions and their adjacency relationship. Segmentation is accomplished when regions is merged in certain principle.

Tradition extraction and mapping method contains visual interpretation, pixel-based classification, artificial neural network classification, reasoning classification of symbolic logic, classification based on knowledge discovery, expert system classification, and so on. Mayunga succeeded in the semi-automatic extraction of buildings using the snake algorithm and radial projection method. Stassopoulou combined canny operator and edge features to recognize buildings under the Baysian network support. Buildings can not be effectively extracted by image classification method because of great disparities in spectral features for buildings in

\footnotetext{
* Corresponding author.
} 
different building material and completion date. Secondly, texture complexity results in inefficiency of texture-based classification.Lastly, rich edge information, complex topological relationships with adjacent objects and shadows, all of these elements lead to no unified classification method efficient for various image. In object-oriented extraction method, the spectral attributes, such as homogeneity, contrast, angular second moment, entropy, correlation, standard deviation and adjacent boundary length are features to be introduced in objectoriented extraction based on the following improved image segmentation.

\section{IMAGE SEGMENTATION THEORY}

\subsection{Image Preprocessing}

There exists certain kind of distortion for satellite image from real earth surface due to sensor, time phase, and projection method factors. Image preprocessing procedures including calibration, registration, mosaic, radiometric correction and denoising are required before further image processing steps. Image material in the thesis has been ortho-rectified and registrated, thus the emphasis focuses on image enhancement to raise contrast of target and background. Image enhancement contains methods such as histogram equalization, sharpening, and gray stretching.

\subsection{Traditional Image Segmentation Method}

Image segmentation is a technique to divide the image into subareas which is related to object and region in earth surface according to certain principle. Segmentation is the basis of object expression and feature measurement. Pixels within the same subarea have similar features while pixels belong to different subareas have different features. Subareas satisfy the following qualification: homogeneity and connectivity, connectivity means there exists connected path between any two pixels.

Traditional segmentation methods conclude as four categories: (1) Threshold segmentation, it has simple theory and computation. Single threshold segmentation divides the image into object and background. When the image is composed of several objects, we need multi-threshold segmentation. Frequently used algorithms are minimum error method introduced by Kitter and maximum class variance method by Ostu. Fig.2 is iterative threshold segmentation result of Fig.1 and Fig.3 is ostu segmentation result of Fig.1. Threshold segmentation method has shortcomings such as difficult selection of threshold and keenness to noises.

(2) Edge based segmentation, edge often has discrete gray level or great feature difference. This kind of method accords with human cognition but is keen to noises. Parallel boundary and serial boundary technique are common. Parallel boundary method detects all marginal points and seeks to connect all the points to form a edge, there are orthogonal gradient operator (Roberts, Prewitt, Sobel), differential operator (Krish) and second derivative operator (Laplacian, Marr-Hildreth, Canny). Corresponding results are as Fig.4, 5, 6, 7.

(3) Region based segmentation, region growing and region splitting and merging are mentioned. The focus of region growing method is to gather similar pixels to form region, and seed region is surrounded by neighbor point. Region growing method works well when the internal of region is uniform and object has connectivity. Corresponding results are as Fig.8.

(4) Specific segmentation, vast theory such as fuzzy set theory, neural network theory, wavelet theory, genetic algorithm, MRF method, mean shift method and mathematical morphology method are introduced into the segmentation research. MRF segmentation has results as Fig.9.

\subsection{Object-oriented Multi-scale Segmentation Method}

Multi-scale segmentation uses a series of scales to form polygon hierarchical network with minimum homogeneity and maximum heterogeneity. In the network of polygon object, the attribute of every image object not only relates to adjacent object but also contains the relationship between sub object and parent object.

Segmentation parameters mainly include scale, band weight, spectral and shape factor. Scale is the threshold for object visual size, and it determines the size of minimum polygon. The scales for extracting buildings and trees are much lower than that of forests. Band weight is related to interesting areas, for example vegetation objects in color infrared images is sensitive to near infrared wave band, less sensitive to green wave. Typically, the spectral information is more important, however, the shape factors complement the segmentation to improve the classification accuracy. For example, the spectral information of buildings and roads are similar, then shape factor helps the separation from each other.

Multi-scale segmentation method includes random field model based segmentation, mean shift based segmentation and virtual fractal net evolution based segmentation. Virtual fractal net evolution segmentation uses the fuzzy subset theory to extract specific object, with large scale object and small scale object both existing in the same space. FNEA segmentation begins with single pixel, the pixel is merged with adjacent pixels with object minimum heterogeneity principle, and the difference between two adjacent objects are made by heuristic processing. Since Baaz and schape introduced local best fit method in 2000, FNEA method has been widely used for object oriented image analysis.

Shape heterogeneity is composed of two parameters, that is, compactness and smoothness. Compactness is shown in Eqs.(1). Smoothness is shown in Eqs.(2).

$$
c=\frac{l}{\sqrt{n}}
$$

Where: 1 is object boundary length(i.e, perimeter), $\mathrm{n}$ is the square root of object size.

$$
h=\frac{l}{b}
$$

Where: 1 is object boundary length(i.e, perimeter), $b$ is the perimeter of minimum bounding rectangle.

Spectral heterogeneity is computed as Eqs.(3), The heterogeneity $f$ can be expressed as Eqs.(4).

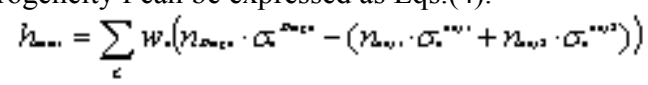

Where: $\sigma_{\mathrm{c}}{ }^{\mathrm{obj} 1}$ is the spectral standard deviation of the first object, $\sigma_{\mathrm{c}}{ }^{\mathrm{obj} 2}$ is that of the second object, $\sigma_{\mathrm{c}}{ }^{\text {Merge }}$ is that of the merged object, $n_{\text {Merge }}$ is the pixel number of the merged object, $n_{\mathrm{obj} 1}$ is that of the first object, $n_{\mathrm{obj} 2}$ is that of the second object

$$
f=w \cdot h_{e d t o r}+(1-w) \cdot h_{\text {shd }}
$$

Where: $h_{\text {color }}$ is spectral heterogeneity and $h_{\text {shape }}$ is shape heterogeneity, the weight $\mathrm{w}_{\text {color }}, \mathrm{w}_{\text {shape }}$, and $\mathrm{w}_{\text {color }}+\mathrm{w}_{\text {shape }}=1$. 
Region merging follows primitive segmentation, and it utilizes the seed point to produce growing area. The growing area follows local or global optimality theory.

The FNEA technique applies local optimality theory to control region merging, it uses the dither matrix generated by binary counter to choose the first object to be merged, promising this object is farthest from the experimented object and distributing the object equally. Specific procedure goes like this: object A and object $\mathrm{B}$ have the minimum heterogeneity growth, at the same time object $\mathrm{C}$ and object $\mathrm{B}$ has the minimum heterogeneity growth, if object $\mathrm{A}$ is the same as object $\mathrm{C}$, local optimality comes true, otherwise, we need to assign object $\mathrm{B}$ to object $A$, object $C$ to object $B$ until all objects are searched.

The multi-scale segmentation procedure is shown as Fig.10. The object hierarchy system is built through the segmentation. Object levels with small scales have rich physical information and less spatial and semantic information, leading to easier extraction of complex surface features. Object levels with big scales are suitable for extraction of simple features.

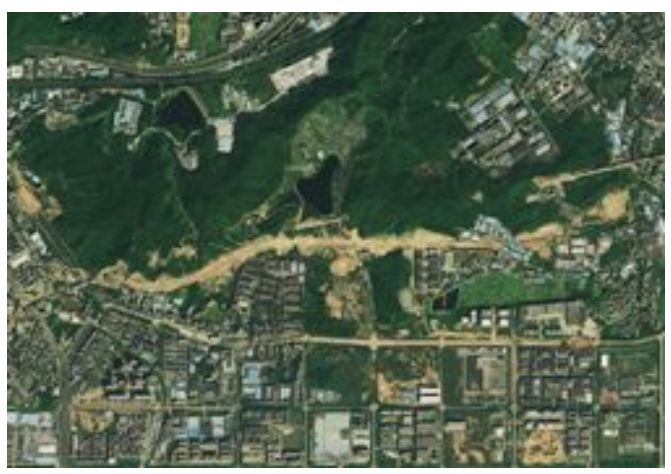

Figure 1. Pleiades image in 2012

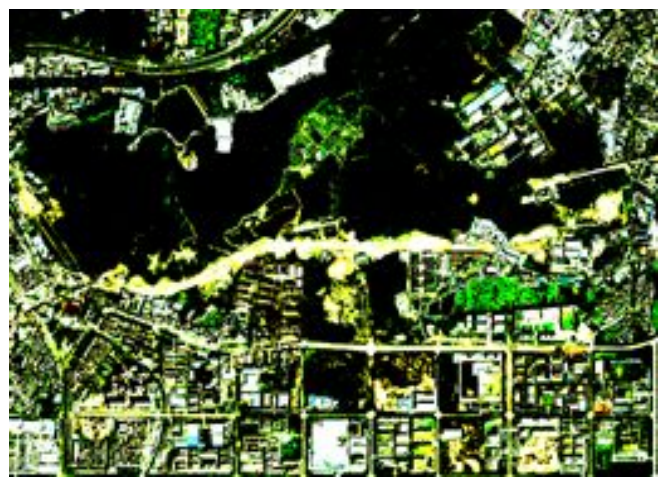

Figure 2. Iterative threshold segmentation

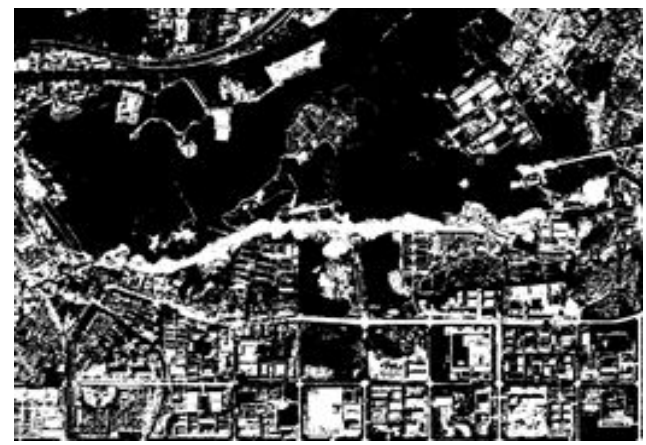

Figure 3. Ostu segmentation

\section{IMPROVED MULTI-SCALE SEGMENTATION METHOD}

\subsection{Improved Watershed Segmentation Algorithm}

The research focuses on a algorithm to gain large area with high internal heterogeneity. Regarding the image as a Region Adjacency Graph formed by the topological relationship between regions. Each initial region connects with neighbor region in 4-direction connectivity rule, and the heterogeneity coefficient can be calculated by spectral and shape feature.

The improved watershed segmentation algorithm orders the pixels in ascending gray value sequence, then fills according to fast breadth first scanning rules. The algorithm goes like this:

(1) Calculate the horizontal and vertical gradient for input image;

(2) Remove irrelevant minimum areas through median filtering and morphological operations on the gradient image;

(3) Order pixel gray value, and store pixels of the same gray value in a queue with pointers to these queue nodes;

(4) The initial water level has the smallest gradient value. If the identified gradient value is higher than $h$, push the pixels in $\mathrm{h}+1$ level which are adjacent to identified pixel in the queue. Scan minimal region in the region, and calculate the area of neighboring region. Merge the regions when the area and gradient difference are lower than the threshold.

(5) If the four neighbor pixels of a pixel have least two identified pixels, this pixel belongs to the watershed, otherwise, only one pixel is marked, label the pixel as the neighbor number and delete the pixel. Process each pixel until the queue is empty, then a new basin is formed.

\subsection{Region Merging Strategy}

Watershed segmentation is followed by region merging. Region merging procedure goes like this:

(1) Traverse the initial regions of watershed segmentation, and calculate the minimum area $\mathrm{C} 1$ of regions;

(2)Find region A whose area is not more than $\mathrm{C} 1$, and search the neighborhood region according to region adjacency graph; (3)Calculate the heterogeneity of the regions in (2), preserve the area with minimum heterogeneity $\mathrm{F}$;

(4)If $\mathrm{F}$ is less than the square result of the given heterogeneity, go to step (5), otherwise, do not merge region and go to step(6); (5)Merge region whose area is less than $\mathrm{Cl}$ and its neighborhood region, then update the feature value and adjacency relation;

(6)Recalculate the minimum region area $\mathrm{C} 2$ whose heterogeneity is less than $\mathrm{F}$;

(7)Traverse the object level until C2 is greater than the given scale and output the segmentation result, otherwise, go to (2).

\section{ARCHITECTURE EXTRACTION THEORY AND EXPERIMENT}

\subsection{Architecture Characteristics}

Architecture in high-resolution image usually has the following five characteristics: (1) Polygon features, it may be U shape, T shape, E shape, or convex and parallel lines; (2) Angle features, the angle has properties of translation, rotation and deformation compression, and in the image the angle is often in 90; (3) Shadow features; (4) Gray features, the top of building has uniform gray level which is higher than surrounding area; (5) 
Texture features, residential building has strip and square texture and plants have color texture or white and light texture.

\subsection{General Situation of Research Area}

Shenzhen locates at longitude from $113^{\circ} 46^{\prime}$ to $114^{\circ} 37^{\prime}$ and

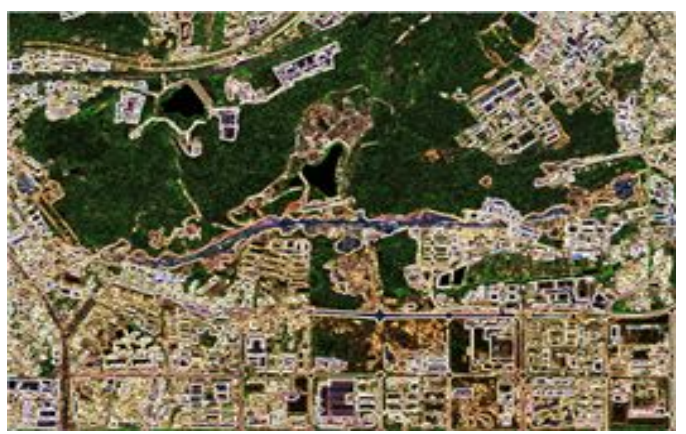

Figure 4. Sobe edge detection



Figure 5. Prewitt edge detection

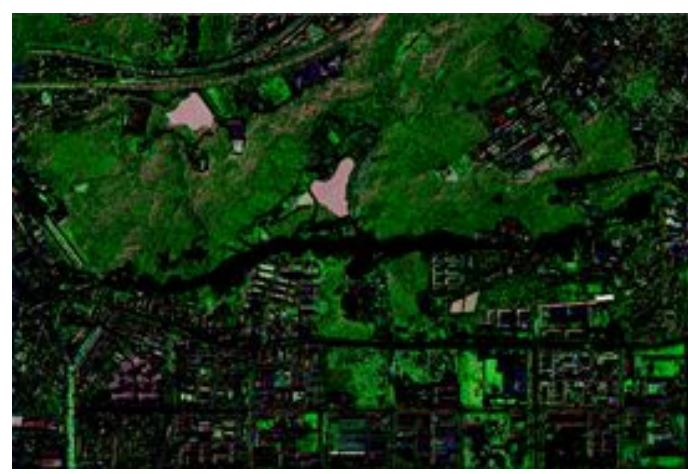

Figure 6. Krish edge detection

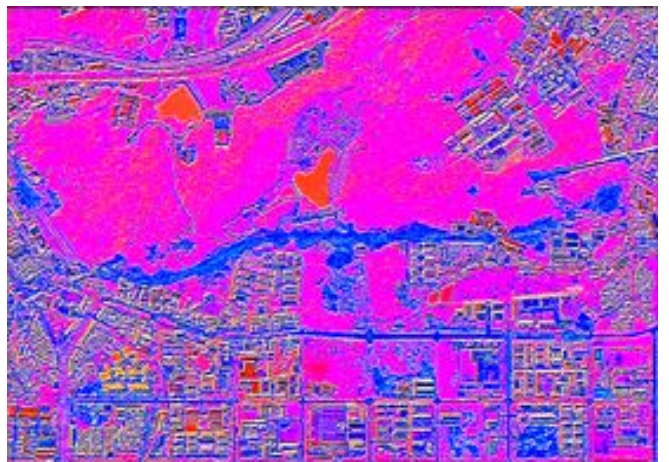

Figure 7. Guass-Laplacian edge detection latitude from $22^{\circ} 27^{\prime}$ to $22^{\circ} 52^{\prime}$. The whole city owns area of

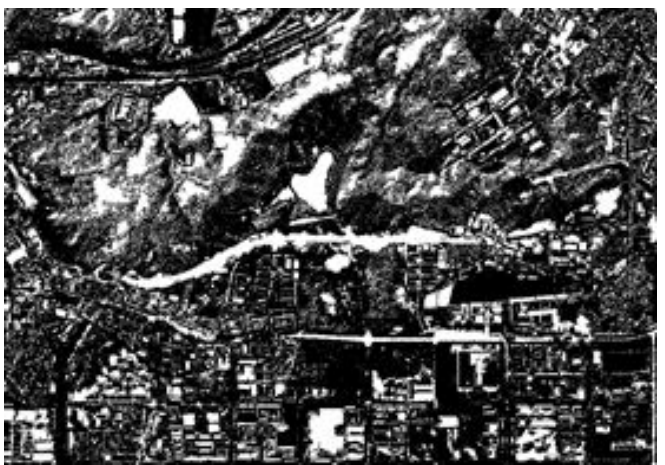

Figure 8. Region growing segmentation

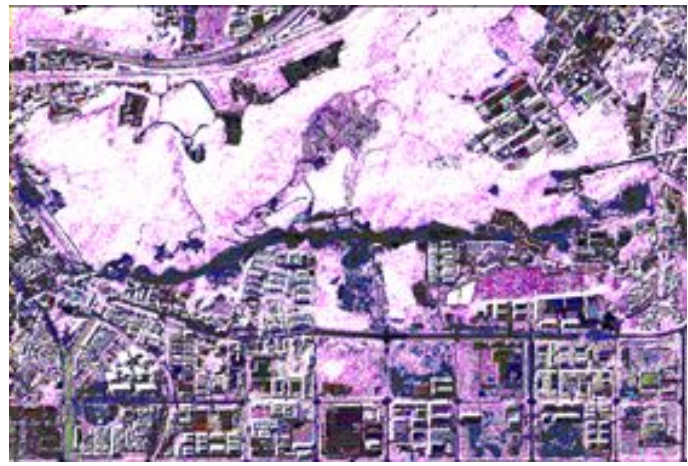

Figure 9. MRF segmentation

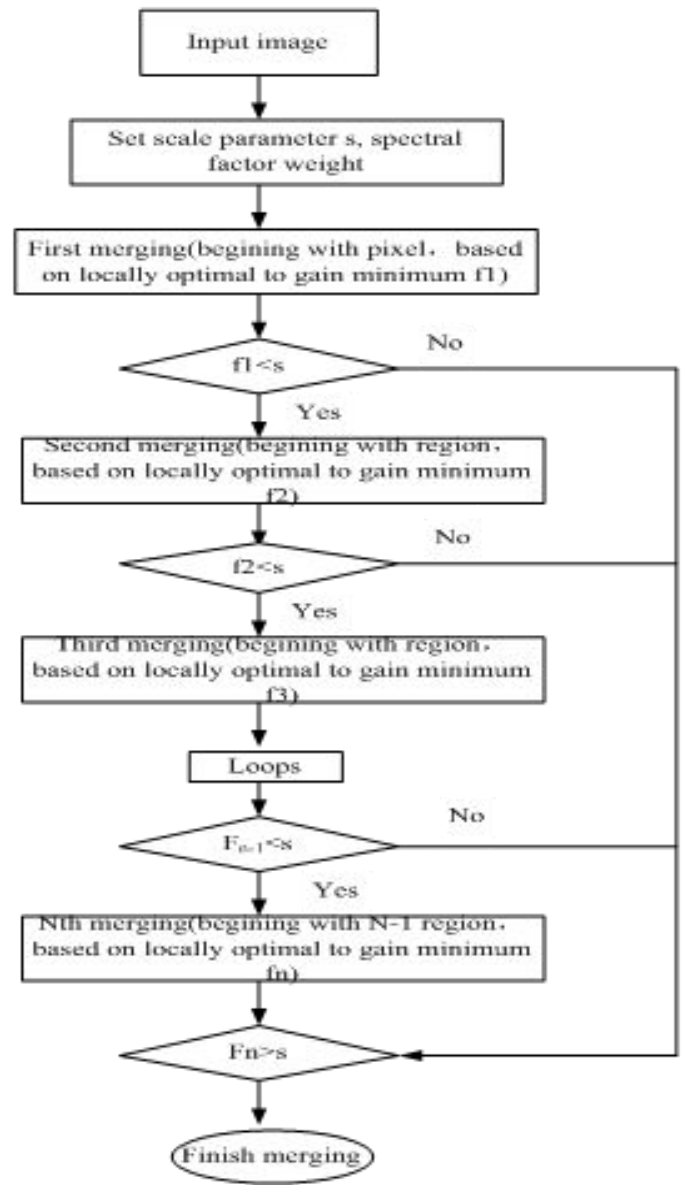

Figure 10. Multi-scale segmentation procedure 


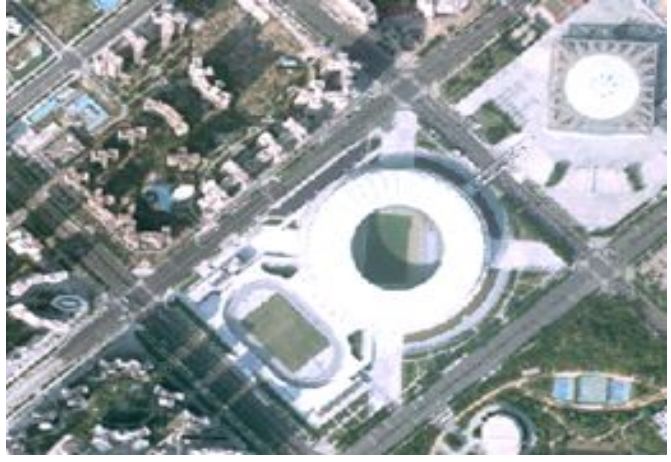

Figure 11. Original pleiades image

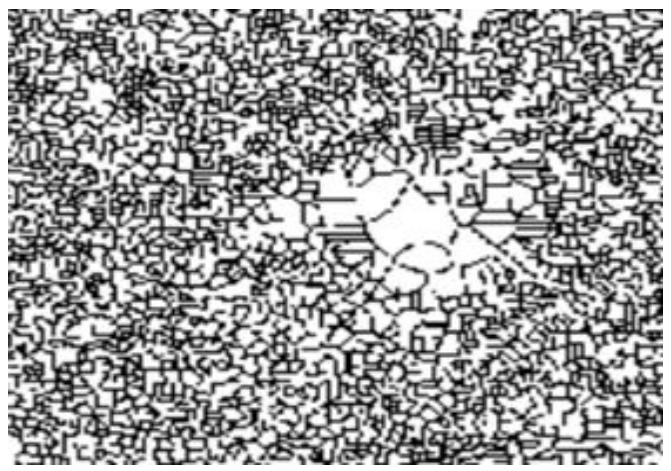

Figure 12. Original watershed segmentation

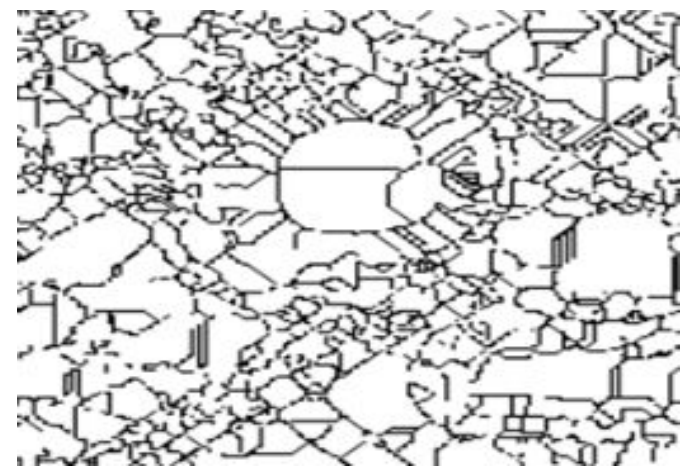

Figure 13. Improved watershed segmentation

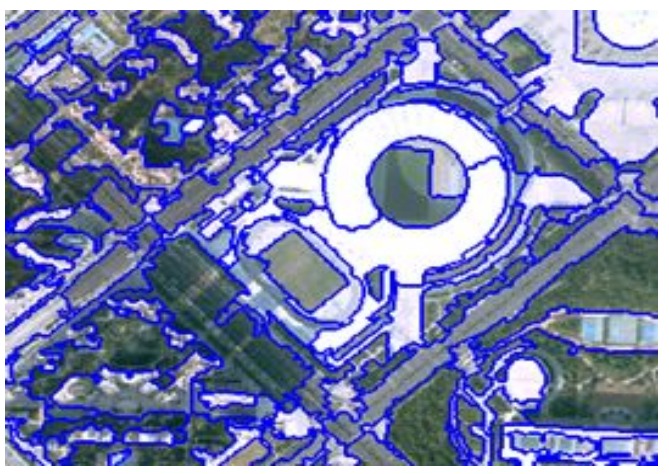

Figure 14. Multi-scale segmentation

1991.64 square kilometres. Shenzhen is a ecological garden city, with $1 / 2$ of the land into basic ecological line of control and no construction is permitted within this region. The application of architecture extraction is used to monitoring the occupation of ecological line.

Experiment data are chosen from Shenzhen Pleiades image in late 2012, as Fig.15 shows. The image owns three bands with resolution of 0.51 meters in BMP format. Image is not affected by cloud and has been done ortho-rectification and geometric correction. Since the image submitted to relevant department has done fusion and color composition, it lacked near infrared spectral band. The thesis mainly studies how to extract architecture with spectral features, geometric features, texture features and spatial relationship in the absence of infrared band.

The size of image is $1010 * 682$. The hardware environment owns CPU of $2 \mathrm{G}$, memory of $2 \mathrm{G}$, and software environment includes Windows XP system, $\mathrm{VC}++6.0$ to run the multi-scale segmentation and extraction algorithm, illustrating results of ENVI 4.5 as comparision and analysis.

\subsection{Architecture Extraction Using Improved segmentation method}

By sampling experimental image object as Table.1, the object repository can be built as Table.2.General characteristics of target images in different periods can be summarized. Then in the establishment of object repository experiment, the multiscale segmentation system is determined. In table1, A means texture, B means homogeneity, C means contrast, D means nonsimilarity, $E$ means average value, $F$ means entropy and $G$ means self-correlation.

According to the best fit scale principle and properties of image, the best fit scale of permanent architecture is 50 . The overall effect of extraction is perfect and prevents the inconsistency with land surface because of difference in area and shape.

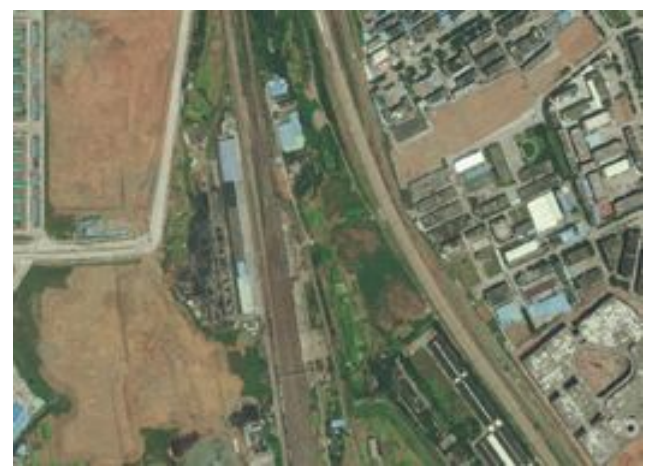

Figure 15. Experimental pleiades image

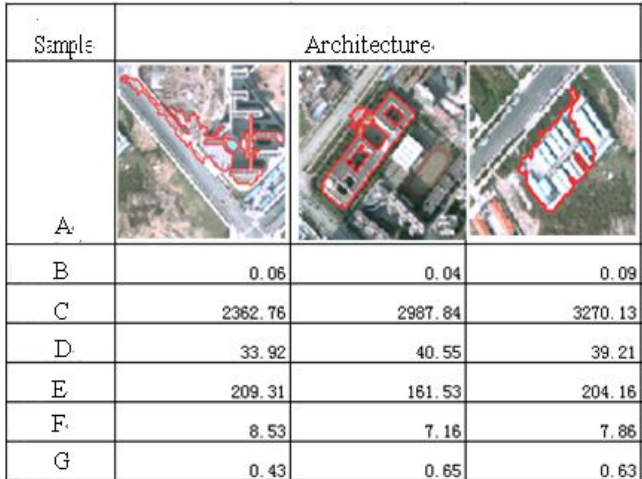

Tabel 1. Object sample 




Table 2. Object repository



Figure16. Permanent architecture extraction

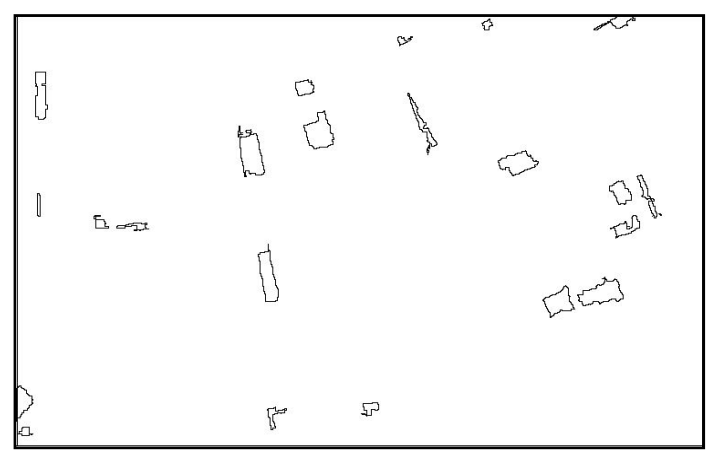

Figure17. Temporary architecture extraction

\subsection{Extraction Accuracy Comparison and Analysis}

Synonyms spectrum phenomenon in traditional extraction method is serious, the salt and pepper always exists, as Fig.18 shows. By comparision, object-oriented extraction produces homogeneous objects, it is more consistent with the real earth surface.

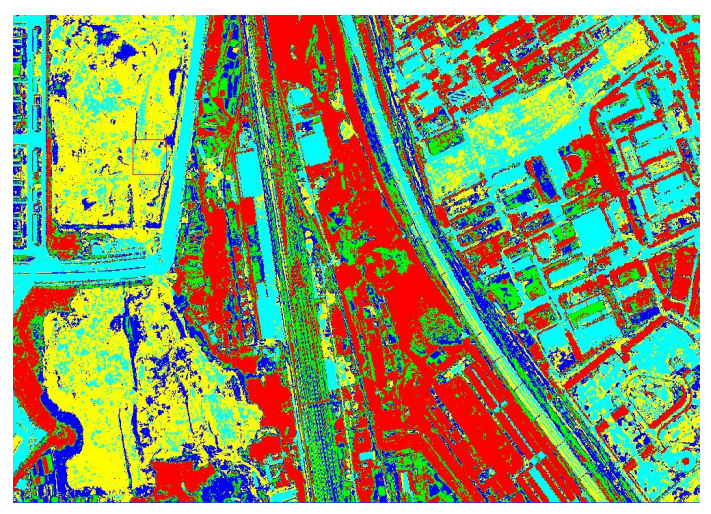

Figure18. K-means extraction result
The research has 258 precision validation sampling points in the scope of the image and carries out classification accuracy verification point by point. According to the existing data and the field investigation and verification, the evaluation results of pixel-based and multi-scale object-oriented segmentation and extraction is shown as Table 3(PA represents permanent architecture, TA represents Temporary architecture). The accuracy of bject-based multi-scale extraction is 11.75 percent higher than that of k-means extraction for permanent architecture and 16.04 percent higher for temporary architecture.

\begin{tabular}{|c|c|c|c|c|c|c|}
\hline Class & base & road & PA & TA & Bare-soil & 0verall \\
\hline base & 30 & 0 & 0 & 0 & 1 & 32 \\
\hline road & 0 & 55 & 1 & 0 & 0 & 56 \\
\hline PA & 0 & 2 & 87 & 0 & 0 & 89 \\
\hline TA & 0 & 0 & 0 & 51 & 1 & 52 \\
\hline $\begin{array}{c}\text { Bare } \\
\text { soil }\end{array}$ & 1 & 0 & 0 & 0 & 28 & 29 \\
\hline Overal1 & 31 & 57 & 88 & 51 & 30 & 258 \\
\hline $\begin{array}{c}\text { Producer } \\
\text { accuracy }\end{array}$ & $96.77 \%$ & $96.49 \%$ & $98.86 \%$ & $\begin{array}{c}100.00 \\
\%\end{array}$ & $93.33 \%$ & \\
\hline $\begin{array}{c}\text { User } \\
\text { accuracy }\end{array}$ & $93.75 \%$ & $98.21 \%$ & $97.75 \%$ & $98.08 \%$ & $96.55 \%$ & \\
\hline $\begin{array}{c}\text { Overal1 } \\
\text { accuracy }\end{array}$ & \multicolumn{7}{|c|}{0.972868217} \\
\hline \begin{tabular}{c} 
Kappa \\
\hline
\end{tabular}
\end{tabular}

Table3. Multi-scale object-oriented extraction accuracy

\begin{tabular}{|c|c|c|c|c|c|c|}
\hline Class & base & road & PA & TA & Bare-soil & 0verall \\
\hline base & 26 & 0 & 0 & 4 & 5 & 40 \\
\hline road & 0 & 50 & 10 & 0 & 1 & 61 \\
\hline PA & 0 & 15 & 67 & 0 & 0 & 82 \\
\hline TA & 3 & 0 & 0 & 35 & 2 & 40 \\
\hline Bare soi1 & 5 & 0 & 0 & 3 & 27 & 35 \\
\hline Overal1 & 34 & 65 & 77 & 42 & 35 & 258 \\
\hline $\begin{array}{c}\text { Producer } \\
\text { accuracy }\end{array}$ & $76.47 \%$ & $76.92 \%$ & $87.01 \%$ & $83.33 \%$ & $77.14 \%$ & \\
\hline $\begin{array}{c}\text { User } \\
\text { accuracy }\end{array}$ & $65.00 \%$ & $81.97 \%$ & $81.71 \%$ & $87.50 \%$ & $77.14 \%$ & \\
\hline $\begin{array}{c}\text { Overal1 } \\
\text { accuracy }\end{array}$ & \multicolumn{7}{|c|}{0.794573643} \\
\hline Kappa & \multicolumn{7}{|c|}{0.737139562} \\
\hline
\end{tabular}

Table 4. K-means segmentation and extraction accuracy

\section{CONCLUSION}

High resolution image has rich information and the segmentation and extraction process for this kind of image offer more feature information for further analysis and recognition. The thesis introduces new improved algorithm for the objectoriented segmentation and extraction of architecture to meet the challenge of remote sensing monitoring tasks. The research findings are: (1) Implement a multi-scale segmentation method based on improved watershed algorithm and region merging algorithm; (2) Make analysis of architecture characteristics and carry out the feature extraction experiment based on the improved segmentation method and tradition extraction method 
to verify the effectiveness and robustness of the improved multi-scale object-oriented segmentation and extraction.

\section{REFERENCES}

Nalwa. On Detecting Edges[J]. IEEE Transactions on Pattern Analysis and Machine Intelligence, 1986, 8(6): 699-714.

Haralick. Morphologic edge detection[J]. IEEE Journal of Robotics and Automation, 1987, 3(2): 142-156.

Staib. Boundary finding with prior shape and smoothness models[J]. IEEE Transactions on Pattern Analysis and Machine Intelligence, 2000, 22(7): 738-743.

Kass. Snakes: Active contour models[J]. International Journal of Computer Vision, 1988, 4(1): 321-331.

Auethavekiat. A two-stage segmentation algorithm using robust anisotropic region growing[J]. Journal of the Institute of Image Information and Television Engineers, 2003, 57(1): 117-125.

DerivauX. Supervised image segmentation using watershed transform, fuzzy classification and evolutionary computation[J]. Pattern Recognition Letters, 2010, 31(15): 2364 - 2374.

Deng and Manjunath. Contrast-based color image segmentation[J]. Signal Processing Letters, 2004, 11(7): 641644 .

M.Baatz, A.Schape. Multiresolution: an optimization approach for high quality multi-scale image segmentation. In: Strobl.j., Blaschke, T., Griesebner, G. ( Eds ) . Agewandte Geographische Informations-Verarbeitung XIII Wichmann Verlag, Karlsruhe, 2000: 12-23.

Xia, L. A method to improve classification with shape information[J]. International Journal of Remote Sensing, 996, 17: $1473-1481$.

Sanghamitra, Bandypopadhyaya, Ujjwal Maulik. Generic clustering for automatic evolution of clusters and application to image classification[C]. Pattern Recognition, 2002, 35(8): 1197 1208 .

Mind-Der Yang, Yeh-Fen Yang. Genetic algorithm for unsupervised classification of remote sensing imagery[J]. Image Processing: Algorithm and System III Proc. Of SPIE_IS\&T Electronic imaging, 2004, 5298: 395-402.

Gong. P. \& Howarth, P.J. Frequency-based contextural classification and gray-level vector reduction for land-use identification $[\mathrm{J}]$. Photogrammetric Engineering and Remote Sensing, 1990, 56: 67-73.

Ton, J., Sticklen, J., and Jain, A.K. Knowledge-Based Segmentation of Landsat images[J]. IEEE Transactions on Geoscience and Remote Sensing, 1991, 29(2): 224-231.

Lobo A, Chic O and Casterad A.Classification of Mediterranean crops with multi-sensor data: per-pixel versus per-object statistics and image segmentation[J]. International Journal of Remote Sensing, 1996, 17: 2358-2400.

GAO YAN, J.F. MAS et al. Comparision of pixel-based and object-oriented image classification approaches-a case study in a coal fire area, Wuda, Inner Mongolia, China[J]. International Journal of Remote Sensing, 2006, 27(18-20): 4039-4055. 